\title{
Synthesis and Identification of Pentathiepin-Based Inhibitors of Sporothrix brasiliensis
}

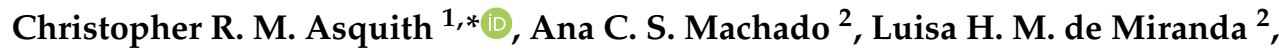 \\ Lidia S. Konstantinova ${ }^{3,4}$, Rodrigo Almeida-Paes ${ }^{5}$ (D), Oleg A. Rakitin ${ }^{3,4}$ (D) and \\ Sandro A. Pereira ${ }^{2}$
}

1 Department of Pharmacology, School of Medicine, University of North Carolina at Chapel Hill, Chapel Hill, NC 27599, USA

2 Laboratory of Clinical Research in Dermatozoonoses on Domestic Animals (LAPCLIN-DERMZOO), Evandro Chagas National Institute of Infectious Diseases (INI), Oswaldo Cruz Foundation (Fiocruz), Rio de Janeiro 21040-360, Brazil; ana.machado@ini.fiocruz.br (A.C.S.M.); luisa.helena@ini.fiocruz.br (L.H.M.d.M.); sandro.pereira@ini.fiocruz.br (S.A.P.)

3 Zelinsky Institute of Organic Chemistry, Russian Academy of Sciences, Moscow 119991, Russia; konstantinova_ls@mail.ru (L.S.K.); orakitin@ioc.ac.ru (O.A.R.)

4 Nanotechnology Education and Research Center, South Ural State University, Lenina Ave. 76, Chelyabinsk 454080, Russia

5 Mycology Laboratory, INI, Fiocruz, Rio de Janeiro 21040-360, Brazil; rodrigo.paes@ini.fiocruz.br

* Correspondence: chris.asquith@unc.edu; Tel.: +1-919-962-5349

Received: 13 November 2019; Accepted: 30 November 2019; Published: 3 December 2019

\begin{abstract}
Sporothrix brasiliensis is the causative agent of zoonotic sporotrichosis in Brazil and is currently referred to as the most virulent species among those of clinical importance within the genus. Sporotrichosis is an emergent disease that has come to the forefront over two decades with a recent hot spot of sporotrichosis infection emerging in the state of Rio de Janeiro. The source of these infections is now at epidemic proportions with more than 4000 cases reported in Rio de Janeiro, Brazil, alone since 1998. We developed a focused library of a rare pentathiepin ring system and identified a potent substitution pattern that yielded compounds 21 and 22. These compounds were more potent than itraconazole which is the current standard of care for sporotrichosis.
\end{abstract}

Keywords: sporotrichosis; 1,2,3,4,5-pentathiepin; varacin; anti-fungal; sulfur heterocycle

\section{Introduction}

Sporotrichosis is an emergent disease that has come to the forefront over two decades after being first described in 1898 [1,2]. A recent hot spot of Sporothrix infection has emerged in the state of Rio de Janeiro, Brazil [3]. The source of these infections is now at hyperendemic proportions with more than 4000 cases reported in this region since 1998 [3-5].

Sporothrix species are usually non-pathogenic environmental fungi that are closely related to decaying wood, plants, and soil [6]. The majority of human and animal infections occur when the epidermis is damaged, allowing plant matter/soil, along with the fungus, to enter into the body [7]. The incidence of zoonotic infection is on the rise with feline transmission of Sporothrix which has been identified as the likely source of the spike in cases within Rio de Janeiro $[3-5,8]$. Sporothrix brasiliensis has been demonstrated to be a highly successful mammal pathogen, and it is related to zoonotic transmission from infected cats in Brazil [9].

Feline sporotrichosis has a varied range of clinical presentation, but it is frequently a severe condition with the development of disseminated skin lesions and respiratory involvement in infected 
cats [10]. Although human sporotrichosis is not usually severe, disseminated and life-threatening cases have been described in association to $S$. brasiliensis infection, especially in immunocompromised patients [11].

Infected cats are the most important source of Sporothrix transmission in the Brazilian scenario due to the high burden of yeasts in their lesions. Zoonotic transmission usually occurs through their scratches and bites $[3,10]$. Although the treatment of feline sporotrichosis is shown to efficiently reduce the fungal burden in lesions of cats contributing to the control of zoonotic transmission, it remains a challenge for veterinary practitioners [12]. Itraconazole frequently in association to potassium iodide is still the treatment of choice, but therapeutic failure, the occurrence of adverse effects, and recrudescence of lesions are described, raising the need for alternative therapeutic options [10].

Several small anti-fungal molecules (1-8) have been reported (Figure 1), targeting different routes to treat fungal infections. The fungal cells, unlike mammalian cells, are encased in a carbohydrate-containing cell wall which has been used as a target to reduce mammalian toxicity and to target specifically the fungus [13]. The fungal cell wall has been targeted with a series of chalcone and quinoline derivatives (1-3) with varying degrees of success [14-16].<smiles>O=C(/C=C/c1ccc([N+](=O)[O-])cc1)c1ccccc1</smiles>

1<smiles>C=CCC(Nc1ccc(Br)cc1)c1ccccc1</smiles>

2<smiles>Cc1ccc2nc(-c3ccccc3)cc(C)c2c1</smiles>

3<smiles>S=c1cc(Sc2ccccc2)ss1</smiles>

4<smiles>N#Cc1snc2c1SSSSS2</smiles>

5

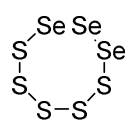

6<smiles>c1ccc(S[Ge]2(O[n+]3ccccc3)Oc3cccc[n+]3S2)cc1</smiles>

7<smiles>CCC(C)n1ncn(-c2ccc(N3CCN(c4ccc(OCC5CO[C@@](Cn6cncn6)(c6ccc(Cl)cc6Cl)O5)cc4)CC3)cc2)c1=O</smiles>

Figure 1. Previously reported anti-fungal compounds.

As part of those screening efforts, a series of 5-(phenylthio)-3H-1,2-dithiole-3-thione derivatives (4) was also identified, where additional sulfur substitutions improved potency [17]. This was supported by a previous report by DuPont in 1977 [18]. In this program, 7 -cyano-7H-(1,2,3,4,5) pentathiepino(6,7-c)pyrazole (5) was developed as a potent anti-fungal in multiple crop species, providing near total control of all fungal growth [18-23]. Two of the three main compounds commercially used to suppress fungal growth are the polysulfide-containing compounds selenium sulfide (6) and pyrithione zinc (7), both used for topical applications (used to treat skin and crops respectively) [24-27].

A major interest of our work has been the reactivity of internal disulfide bridge compounds and their medicinal chemistry applications [28-38]. The high-density polysulfide heterocycles lend themselves to this type of redox-type applications including zinc ejection (in multiple systems) [29-37], anti-viral [29-35], cancer [36,37], and oxidative stress [38].

The pentathiepin functionality $(\mathbf{5}, \mathbf{9 - 1 7})$ has been utilized for several different indications including neoplastic diseases, Alzheimer's, viruses, cancer, bacteria, mycoses, and as an inhibitor of protein kinase C (Figure 2) [18-23,32,39-50]. The DuPont compound $\mathbf{5}$ showed promising activity against fungus in plants with broad spectrum activity against a wider range of plant disease [18]. While the natural product varacin (9) showed potent inhibition of Candida albicans at a rate $100 \times$ that of 
5-fluorouracil [39]. More recently, compound $\mathbf{1 7}$ has shown good inhibition activity against S. aureus,

C. albicans, and C. neoformans with an impressive toxicity profile [50].

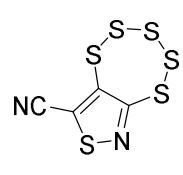

5

Anti-Fungal

1977

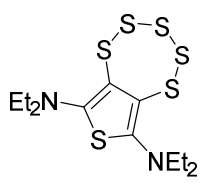

13

Anti-Viral

2014<smiles>COc1cc(CCN)c2c(c1OC)SSSSS2</smiles>

Varacin - 9

Anti-Fungal

1991

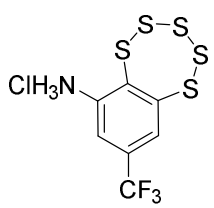

14

Anti-Alzheimers

2015

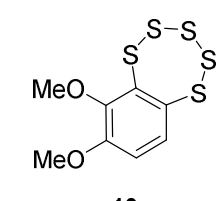

10

Anti-Cancer

1995

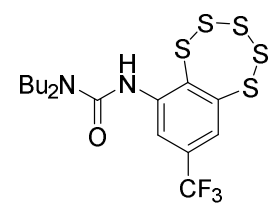

15

Anti-Cancer

2015
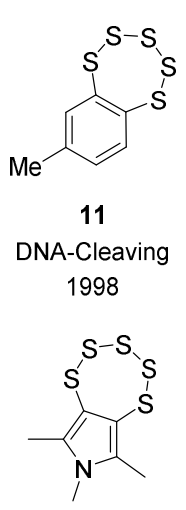

16

Anti-Viral

2018
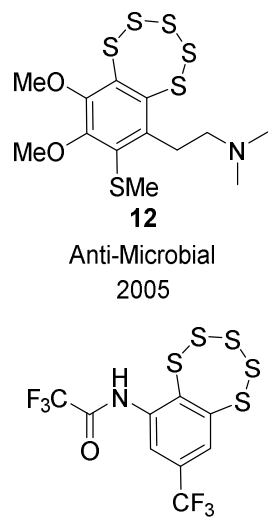

17

Anti-Microbial

2019

Figure 2. Previously reported biologically active pentathiepin compounds.

\section{Results}

\subsection{Initial Investigation}

To investigate pentathiepin as a viable treatment for sporotrichosis, we first screened a symmetric thiophene derivative (13) against six isolates of S. brasiliensis from skin lesions of infected cats. The antifungal susceptibility testing was based on the reference broth microdilution method according to the M38-A2 CLSI guidelines [51] with a few modifications to improve minimal inhibitory concentration (MIC) determination for S. brasiliensis [52]. We screened 13 at two concentrations $(4$ and $8 \mu \mathrm{g} / \mathrm{mL})$ to determine if there was any inhibition of fungal growth. We evaluated MIC to suppress both $100 \%$ and $50 \%$ of the growth of the strains. At the higher concentration, 100\% inhibition of growth was observed in all six isolates with the isolate 8607 presenting a slightly higher sensitivity and being the only isolate to respond to this compound at the lower concentration (Table 1).

Table 1. Initial screening of compounds $\mathbf{1 3}$ and $\mathbf{8}$ against six feline isolates of Sporothrix brasiliensis.

\begin{tabular}{cccccccc}
\hline \multirow{2}{*}{ Compound } & $\begin{array}{c}\text { Growth Inhibition } \\
\text { Percent }\end{array}$ & \multicolumn{5}{c}{ Isolates of S. brasiliensis/MIC $(\mu \mathrm{g} / \mathbf{m L})^{\text {a }}$} \\
\cline { 2 - 7 } & $100 \%$ & 8547 & $\mathbf{8 5 8 4}$ & $\mathbf{8 6 0 7}$ & $\mathbf{8 6 1 2}$ & $\mathbf{8 7 7 5}$ & $\mathbf{8 7 2 9}$ \\
\hline \multirow{2}{*}{$\mathbf{1 3}$} & $50 \%$ & $>8$ & $>8$ & 8 & 8 & 8 & 8 \\
& $100 \%$ & 0.5 & 0.5 & 1 & 1 & 2 & 2 \\
\hline $\mathbf{8}$ & \multicolumn{7}{c}{${ }^{a}$ Mean average $(n=4)}$.
\end{tabular}

\subsection{Synthesis of Pentathiepin Analogs}

We decided to prepare a small array of pentathiepins $(\mathbf{1 3}, \mathbf{1 6}$, and 18-24) (Scheme 1). The older methods for preparation of this advanced heterocycle included using an activated sulfur source, such as disulfur dichloride $\left(\mathrm{S}_{2} \mathrm{Cl}_{2}\right)$ or trisulfur dichloride $\left(\mathrm{S}_{3} \mathrm{Cl}_{2}\right)$ or even directly with elemental sulfur $\left(\mathrm{S}_{8}\right)$, and adding these to an ortho-dithiol, usually under harsh conditions [53-57]. However, a $\mathrm{C}-\mathrm{H}$ activated route utilizing the 1,4-diaza-bicyclo(2.2.2)octane (DABCO) sulfur monochloride complex enables formation of pentathiepins in one step from commercially available reagents (Scheme 1) [58,59]. The two thiophene analogs (13 and 18) were furnished by treating Hünig's base and $N, N$-dibenzylethanamine, respectively, with $\mathrm{DABCO}$ and $\mathrm{S}_{2} \mathrm{Cl}_{2}$ for $48 \mathrm{~h}$ followed by refluxing with triethylamine for a further $2 \mathrm{~h}$ to afford 13 and 18 in good and acceptable yields, respectively [32,60]. The pyrrole derivatives were 
explored using a series of substituted pyrrole ring systems and treating with DABCO and $\mathrm{S}_{2} \mathrm{Cl}_{2}$ for 48 hours to access 16, 19, and 20 in good yield [61]. Treatment of $N$-methylpyrrolidine under analogous conditions allowed access to 21 and 23 in a one-pot reaction. However, under the same conditions, $\mathrm{N}$-isopropylpyrrolidine produced exclusively 22 with the corresponding asymmetric product not observed [62-64]. This was followed by a final analog (24) synthesized by treating $N$-methylindole with the DABCO sulfur monochloride complex at zero degrees to furnish the final product in excellent yield $(70 \%)[64,65]$.
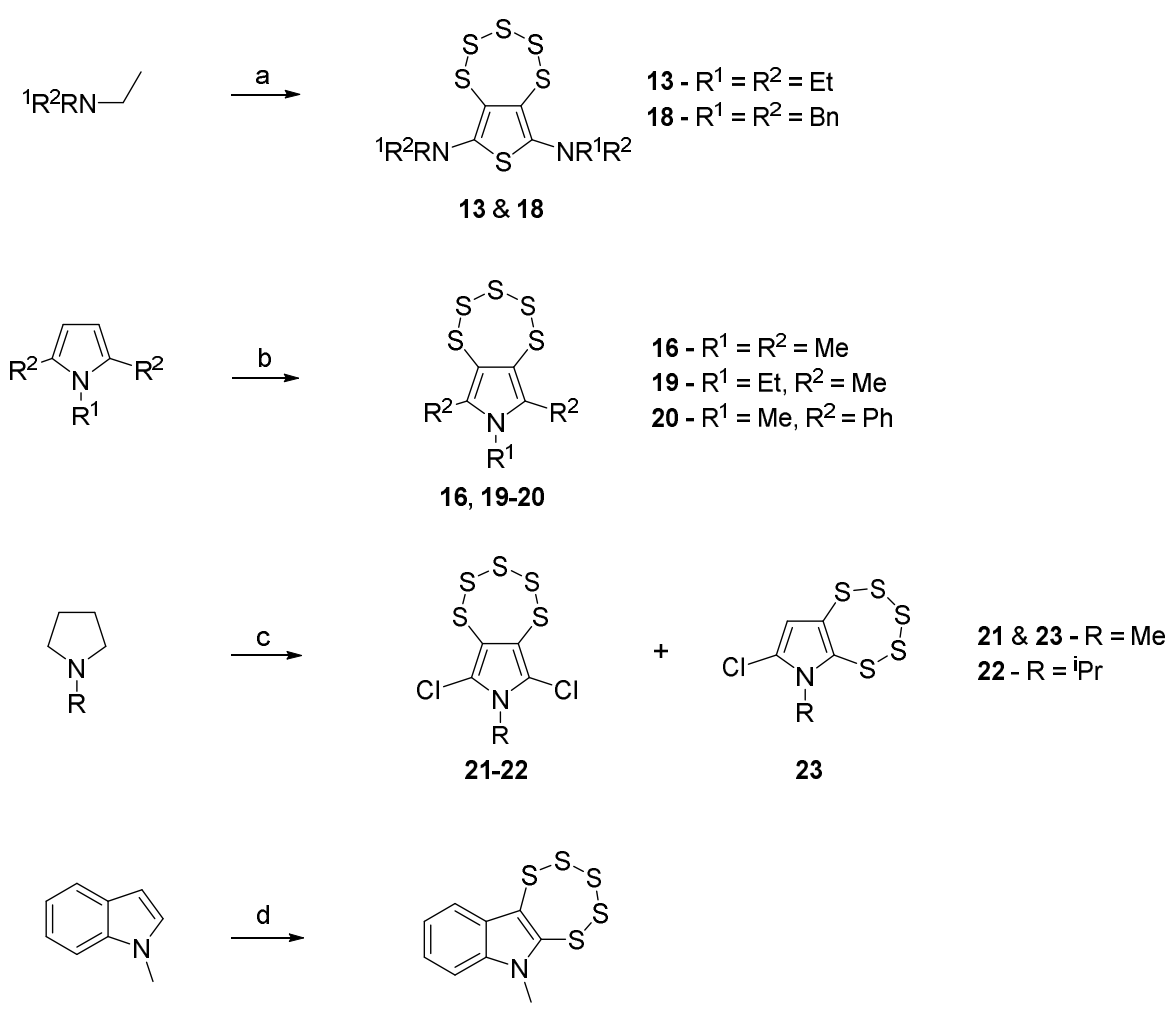

24

Scheme 1. Synthesis route to pentathiepin derivatives: reagents and conditions. (a) 1,4-Diaza-bicyclo (2.2.2)octane (DABCO), $\mathrm{S}_{2} \mathrm{Cl}_{2}, \mathrm{CHCl}_{3}, 0{ }^{\circ} \mathrm{C}$ to rt; $48 \mathrm{~h}$, reflux $2 \mathrm{~h}$ (13,30\% and 18, 3\%); (b) DABCO, $\mathrm{S}_{2} \mathrm{Cl}_{2}, \mathrm{CHCl}_{3}, 0{ }^{\circ} \mathrm{C} ; 48 \mathrm{~h}(\mathbf{1 6}, 36 \% ; \mathbf{1 9}, 40 \%$ and $\mathbf{2 0}, 62 \%)$; (c) DABCO, $\mathrm{S}_{2} \mathrm{Cl}_{2}, \mathrm{CHCl}_{3}, 20{ }^{\circ} \mathrm{C} ; 48 \mathrm{~h}(\mathbf{2 1}, 18 \%$; 23, 38\% and 22,16\%); (d) $\mathrm{S}_{2} \mathrm{Cl}_{2}$ (0.8 eq), $\mathrm{CHCl}_{3}, 0{ }^{\circ} \mathrm{C} ; 48 \mathrm{~h}(\mathbf{2 4}, 70 \%)$.

\subsection{Evaluation of Pentathiepin Analogs}

We then screened this series of symmetrical derivatives $(\mathbf{1 6}, \mathbf{1 8 - 2 2})$ against eight skin lesion isolates of S. brasiliensis (Table 2). We found that increasing the size of the substituent on the amine of the thiophene (18) removed all activity. The 1,2,5-trimethyl-pyrrole (16) was also inactive, with an $N$-ethyl derivative (19) and 2,5-diphenyl derivative (20) also showing no activity. Interestingly, the 2,5-dichloro, $\mathrm{N}$-methyl compound (21) showed some activity on two isolates with the 2,5-dichloro, $\mathrm{N}$-isopropyl (22) extending this to three isolates. 
Table 2. Screening results of a symmetrical pentathiepin array against isolates of Sporothrix brasiliensis.

\begin{tabular}{|c|c|c|c|c|c|c|c|c|c|c|}
\hline \multirow{2}{*}{ Cmpd } & \multirow{2}{*}{$\mathbf{R}$} & \multirow{2}{*}{$\mathbf{X}$} & \multicolumn{8}{|c|}{ Isolates of $S$. brasiliensis/MIC $(\mu \mathrm{g} / \mathrm{mL})^{\text {a }}$} \\
\hline & & & 8547 & 8726 & 8440 & 8607 & 8639 & 8775 & 8729 & 8902 \\
\hline 13 & $\mathrm{~N}-(\mathrm{Et})_{2}$ & S & 8 & nt & nt & 4 & nt & 8 & 8 & nt \\
\hline 18 & $\mathrm{~N}-(\mathrm{Bn})_{2}$ & $S$ & $>8$ & $>8$ & $>8$ & $>8$ & $>8$ & $>8$ & $>8$ & $>8$ \\
\hline 16 & $\mathrm{CH}_{3}$ & $\mathrm{~N}-\mathrm{CH}_{3}$ & $>8$ & $>8$ & $>8$ & $>8$ & $>8$ & $>8$ & $>8$ & $>8$ \\
\hline 19 & $\mathrm{CH}_{3}$ & $\mathrm{~N}-\mathrm{CH}_{2} \mathrm{CH}_{3}$ & $>8$ & $>8$ & $>8$ & $>8$ & $>8$ & $>8$ & $>8$ & $>8$ \\
\hline 20 & $\mathrm{Ph}$ & $\mathrm{N}-\mathrm{CH}_{3}$ & $>8$ & $>8$ & $>8$ & $>8$ & $>8$ & $>8$ & $>8$ & $>8$ \\
\hline 21 & $\mathrm{Cl}$ & $\mathrm{N}-\mathrm{CH}_{3}$ & 8 & $>8$ & $>8$ & $>8$ & 8 & $>8$ & $>8$ & $>8$ \\
\hline 22 & $\mathrm{Cl}$ & $\mathrm{N}-\mathrm{C}\left(\mathrm{CH}_{3}\right)_{2}$ & 8 & $>8$ & $>8$ & $>8$ & 8 & $>8$ & $>8$ & 8 \\
\hline
\end{tabular}

${ }^{a}$ Mean average $(n=4) ; \mathrm{nt}=$ not tested.

Encouraged by our early results, we screened the same set of isolates with two unsymmetrical pentathiepin derivatives (23 and 24) (Table 3). We found antifungal activity across all isolates with both 23 and 24.

Table 3. Results of asymmetric pentathiepins against isolates of Sporothrix brasiliensis.

\begin{tabular}{|c|c|c|c|c|c|c|c|c|c|c|}
\hline \multirow{2}{*}{ Name } & \multirow{2}{*}{$\mathbf{R}$} & \multirow{2}{*}{$X$} & \multicolumn{8}{|c|}{ Isolates of $S$. brasiliensis/MIC $(\mu \mathrm{g} / \mathrm{mL})^{\text {a }}$} \\
\hline & & & 8547 & 8726 & 8440 & 8607 & 8639 & 8775 & 8729 & 8902 \\
\hline 23 & $\mathrm{Cl}$ & $\mathrm{N}-\mathrm{CH}_{3}$ & 1 & 1 & 1 & 1 & 0.5 & 0.5 & 1 & 1 \\
\hline 24 & & $\mathrm{~N}-\mathrm{CH}_{3}$ & 2 & 4 & 4 & 2 & 2 & 2 & 2 & 2 \\
\hline \multicolumn{3}{|c|}{ Itraconazole (8) } & 0.5 & 0.5 & 0.5 & 1 & 1 & 2 & 2 & 2 \\
\hline
\end{tabular}

${ }^{\text {a }}$ Mean average $(n=4)$.

Compound 23 presented a lower MIC in comparison to itraconazole (8) in 4/8 cases with the others matching or half as effective. Compound 24 was slightly less active, matching $3 / 8$ and 1 or 2 fold less effective in the other $5 / 8$ isolates. Compound 24 demonstrated a robust dose-dependent inhibition of $S$. brasiliensis growth. This can be observed in Figure 3 with decreasing concentrations $(0.5$ to $0.015 \mu \mathrm{g} / \mathrm{mL}$ ) of $\mathbf{2 4}$ having a significant impact on growth of Sporothrix brasiliensis.

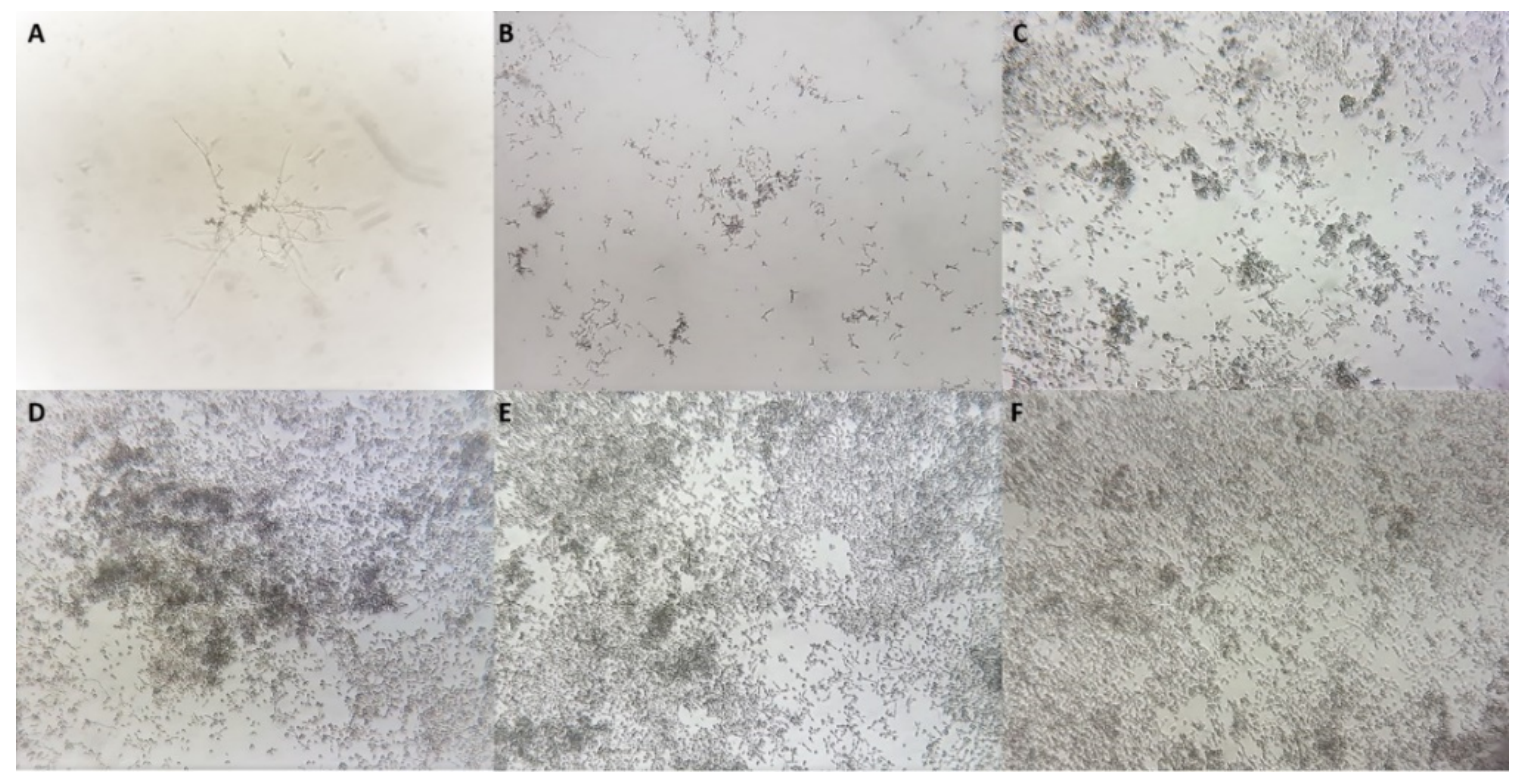

Figure 3. Growth of Sporothrix brasiliensis isolate 8584 treated with compound 24 at (A) $0.5 \mu \mathrm{g} / \mathrm{mL}$ $(0.172 \mu \mathrm{M})$; (B) $0.25 \mu \mathrm{g} / \mathrm{mL}(0.086 \mu \mathrm{M})$; (C) $0.12 \mu \mathrm{g} / \mathrm{mL}(0.041 \mu \mathrm{M})$; (D) $0.06 \mu \mathrm{g} / \mathrm{mL}(0.020 \mu \mathrm{M})$; (E) $0.03 \mu \mathrm{g} / \mathrm{mL}(0.010 \mu \mathrm{M})$; (F) $0.015 \mu \mathrm{g} / \mathrm{mL}(0.005 \mu \mathrm{M})$. 


\section{Discussion}

Sporotrichosis is still a neglected disease. It has reached a significant number of cases in Rio de Janeiro region due to the fact of its uncontrolled zoonotic spread from infected cats. However, not only has the expansion of the disease been identified in other regions in Brazil and Argentina, worldwide emergence is clear, whether related or not to zoonotic transmission. Outbreaks have been described on different continents and infected immunosuppressed patients are at serious risk. In addition, Sporothrix brasiliensis is the causative agent of zoonotic sporotrichosis in Brazil and is currently referred to as the most virulent species among those of clinical importance within the genus.

The sub-micro molar potencies of the pentathiepins coupled with previous reports of moderate toxicities suggests a low-risk path towards the development of a candidate compound for targeting sporotrichosis in cats and potentially humans [32]. The two asymmetric pentathiepins (23 and 24) both show improvements over the current standard of care (itraconazole). While the symmetrical analogs showed only weak activity, increasing the electron withdrawing nature of the substituents (methyl (16) versus chloro (21)) did increase anti-fungal potency. There is increasing evidence that the pentathiepin functionality itself is not a toxic motif and that rather the electronic contributions pendant arms are what drives non-specific toxicity. The pentathiepin functionality and other high-density sulfur heterocycles provide an exciting opportunity for the development of new clinically relevant compounds highlighted by $\mathbf{2 3}$ and $\mathbf{2 4}$. The pentathiepin functionality, while still under-explored, has the potential to generate a pre-clinical candidate for treating sporotrichosis in vivo.

\section{Materials and Methods}

\subsection{Isolate Collection}

The isolates were previously collected from cats with sporotrichosis seen at the Laboratory of Clinical Research on Dermatozoonosis in Domestic Animals (Lapclin-Dermzoo), Evandro Chagas National Institute of Infectious Diseases (INI), Oswaldo Cruz Foundation (Fiocruz), Rio de Janeiro, Brazil. The strains were initially recovered from the exudate of ulcerated lesions or secretions from nasal cavities that were collected using a sterile swab and cultured on Sabouraud dextrose agar and Mycobiotic Agar (Difco), incubated at $25^{\circ} \mathrm{C}$ and observed over 4 weeks for fungal growth. Suspected isolates were sub-cultivated on brain heart infusion agar medium (Difco) at $37^{\circ} \mathrm{C}$, and dimorphism was demonstrated by conversion to the yeast-like form. Then, the isolates were stored in sterile distilled water in the Mycology Laboratory of INI until used.

\subsection{General Procedures for Screening}

The compounds $(\mathbf{1 3}, \mathbf{1 6}, \mathbf{1 8}-\mathbf{2 4})$ were tested using the broth microdilution technique in accordance with the respective reference protocols of the CSLI (Clinical and Laboratory Standards Institute). The reading of the plates was performed in wells with $100 \%$ inhibition of fungal growth (all isolates). All isolates were previously identified by the T3B-fingerprint technique as S. brasiliensis, and their antifungal susceptibilities to itraconazole are known [66]. Repetitions of the test were performed to confirm each result.

\subsection{Chemistry}

\subsubsection{General Procedure to Afford Compounds $\mathbf{1 3}$ and $\mathbf{1 8}$}

Disulfur dichloride $(100 \mathrm{mmol})$ was added dropwise at $-15^{\circ} \mathrm{C}$ to $-20^{\circ} \mathrm{C}$ to a stirred solution of $\mathrm{DABCO}(100 \mathrm{mmol})$ in chloroform $(40 \mathrm{~mL})$. The mixture was stirred at $0^{\circ} \mathrm{C}$ for $48 \mathrm{~h}$. The corresponding amine (200 mmol) was added, and the mixture was refluxed for $2 \mathrm{~h}$, filtered, and the solvents were evaporated. The residue was separated by column chromatography (light petroleum and then light petroleum- $-\mathrm{CH}_{2} \mathrm{Cl}_{2}$ mixtures) to afford products $\mathbf{1 3}$ and $\mathbf{1 8}$. 
6,8-Bis(diethylamino)thieno(3,4-f)(1,2,3,4,5)pentathiepin (13)

Orange oil (1.42 g, 30\%). Anal. Calcd for $\mathrm{C}_{12} \mathrm{H}_{20} \mathrm{~N}_{2} \mathrm{~S}_{6}(\%)$ : C, 37.5; $\mathrm{H}, 5.2 ; \mathrm{N}, 7.3 ; \mathrm{S}, 50.0$ Found (\%) 37.6; H, 5.3; N, 7.3; S, 50.1. ${ }^{1} \mathrm{H} \mathrm{NMR}\left(300 \mathrm{MHz}, \mathrm{CDCl}_{3}\right) \delta: 3.17\left(\mathrm{q}, 8 \mathrm{H}, \mathrm{J}=7.2,4 \times \mathrm{CH}_{2}\right), 1.11(\mathrm{t}, 12 \mathrm{H}, \mathrm{J}=$ 7.2, $\left.4 \times \mathrm{CH}_{3}\right) ;{ }^{13} \mathrm{C} \mathrm{NMR}\left(75.5 \mathrm{MHz}, \mathrm{CDCl}_{3}\right) 152.9$ and $125.1\left(2 \times \mathrm{sp}^{2}\right.$ tertiary $\left.\mathrm{C}\right), 50.6\left(\mathrm{CH}_{2}\right), 13.0\left(\mathrm{CH}_{3}\right)$. $\mathrm{IR},(\mathrm{KBr}) v / \mathrm{cm}^{-1}: 2970(\mathrm{CH}), 1500,1440,1380,1240,1180,1130,1090,840 ; \mathrm{m} / \mathrm{z}(\mathrm{EI}) \mathrm{m} / \mathrm{z} 384\left(\mathrm{M}^{+}, 46 \%\right)$, $320\left(\mathrm{M}^{+}-\mathrm{S}_{2}, 100 \%\right), 287(65), 259(95), 244(65), 231(69) . \mathrm{C}_{12} \mathrm{H}_{20} \mathrm{~N}_{2} \mathrm{~S}_{6}$ requires $\left(\mathrm{M}^{+}\right)$383.9951, found $\left(\mathrm{M}^{+}\right)$383.9943. Consistent with a previous report [60].

\section{6,8-Bis(dibenzylamino)thieno(3,4-f)(1,2,3,4,5)pentathiepin (18)}

Orange oil (1.68 g, 21\%). Anal. Calcd for $\mathrm{C}_{32} \mathrm{H}_{28} \mathrm{~N}_{2} \mathrm{~S}_{6}$ (\%): C, 60.7; H, 4.5; N, 4.4 Found (\%) C, 60.4; $\mathrm{H}, 4.2 ; \mathrm{N}, 4.8 .{ }^{1} \mathrm{H} \mathrm{NMR}\left(300 \mathrm{MHz}, \mathrm{CDCl}_{3}\right) \delta: 7.30(\mathrm{~s}, 5 \mathrm{H}, \mathrm{Ph}), 4.22\left(\mathrm{~s}, 2 \mathrm{H}, \mathrm{CH}_{2} \mathrm{Ph}\right) ;{ }^{13} \mathrm{C} \mathrm{NMR}(75.5 \mathrm{MHz}$, $\left.\mathrm{CDCl}_{3}\right) 154.4,136.9$ and $127.1\left(3 \times \mathrm{sp}^{2}\right.$ tertiary $\left.\mathrm{C}\right), 128.6,128.4$ and $127.5(3 \times \mathrm{CH} \mathrm{Ph}), 59.9\left(\mathrm{CH}_{2} \mathrm{Ph}\right) . \mathrm{IR}$, (KBr) $v / \mathrm{cm}^{-1}: 3080,3060,3030$, and $2920(\mathrm{CH}), 1600,1460,1360,1180,1030,910,740 ; \mathrm{m} / \mathrm{z}$ (EI) 275632 $(\mathrm{M}+, 6 \%), 568\left(\mathrm{M}^{+}-\mathrm{S}_{2}, 4 \%\right), 477(28), 356(52), 287(25), 91(100) . \mathrm{C}_{32} \mathrm{H}_{28} \mathrm{~N}_{2} \mathrm{~S}_{6}$ requires $\left(\mathrm{M}^{+}\right) 632.0577$, found $\left(\mathrm{M}^{+}\right)$632.0557. Consistent with a previous report [60].

\subsubsection{General Procedure to Afford Compounds 16 and 19-22}

Disulfur dichloride $(12.5 \mathrm{mmol})$ was added dropwise at $-25^{\circ} \mathrm{C}$ to $-35^{\circ} \mathrm{C}$ to a stirred solution of DABCO (12.5 mmol) in chloroform $(40 \mathrm{~mL})$ under argon. The mixture was stirred at $\mathrm{rt}$ for $1 \mathrm{~h}$. The corresponding substituted 2,5-dimethylpyrrole $(5.0 \mathrm{mmol})$ in chloroform $(10 \mathrm{~mL})$ was added, and the mixture was stirred at $0{ }^{\circ} \mathrm{C}$ for $48 \mathrm{~h}$ under argon, filtered, and the solvents evaporated. The residue was separated by column chromatography (light petroleum and then light petroleum $-\mathrm{CH}_{2} \mathrm{Cl}_{2}$ mixtures) to afford products 16 and $\mathbf{1 9 - 2 2}$.

6,7,8-Trimethyl-7H-(1,2,3,4,5)pentathiepino(6,7-c)pyrrole (16) yellow solid (4.80 g, 36\%) m.p. 157-159 ${ }^{\circ} \mathrm{C}$. Anal. Calcd for $\mathrm{C}_{7} \mathrm{H}_{9} \mathrm{NS}_{5}(\%): \mathrm{C}, 31.47 ; \mathrm{H}, 3.40 ; \mathrm{N}, 5.25$. Found $(\%) \mathrm{C}, 31.75 ; \mathrm{H}, 3.43 ; \mathrm{N}, 5.18$. ${ }^{1} \mathrm{H} \mathrm{NMR}\left(300 \mathrm{MHz}, \mathrm{CDCl}_{3}\right) \delta: 3.40\left(3 \mathrm{H}, \mathrm{s}, \mathrm{CH}_{3}\right), 2.48\left(6 \mathrm{H}, \mathrm{s}, 2 \times \mathrm{CH}_{3}\right) .{ }^{13} \mathrm{C} \mathrm{NMR}\left(75.5 \mathrm{MHz}, \mathrm{CDCl}_{3}\right)$ $135.2\left(2 \times \mathrm{sp}^{2}\right.$ tertiary C), 120.5, $31.8\left(\mathrm{CH}_{3}\right), 11.3\left(2 \times \mathrm{CH}_{3}\right) . \mathrm{IR},(\mathrm{KBr}) \mathrm{v} / \mathrm{cm}^{-1}: 2930(\mathrm{C}-\mathrm{H}), 2860(\mathrm{C}-\mathrm{H})$, 1520, 1430, 1420, 1400, 1370, 1350, 1030, 100, 810, 780. m/z (EI) $267\left(\mathrm{M}^{+}, 25\right), 203$ (100), 170 (60), 64 (26), 56 (64). $\mathrm{C}_{7} \mathrm{H}_{9} \mathrm{NS}_{5}$ requires $\left(\mathrm{M}^{+}\right) 266.9339$, found $\left(\mathrm{M}^{+}\right)$266.9331. Consistent with a previous report [61].

7-Ethyl-6,8-dimethyl-7H-(1,2,3,4,5)pentathiepino(6,7-c)pyrrole (19) yellow solid (5.62 g, 40\%) m.p. 159-160 ${ }^{\circ} \mathrm{C}$. Anal. Calcd for $\mathrm{C}_{8} \mathrm{H}_{11} \mathrm{NS}_{5}(\%)$ : C, 34.17; H, 3.95; N, 4.98 Found (\%) $\mathrm{C}, 34.35 ; \mathrm{H}, 3.86 ; \mathrm{N}$, 5.09. ${ }^{1} \mathrm{H}$ NMR (300 MHz, $\left.\mathrm{CDCl}_{3}\right)$ 8: $3.80\left(2 \mathrm{H}, \mathrm{q}, \mathrm{J}=7.2 \mathrm{~Hz}, \mathrm{CH}_{2}\right) ; 1.28\left(3 \mathrm{H}, \mathrm{t}, \mathrm{J}=7.2 \mathrm{~Hz}, \mathrm{CH}_{3}\right) .{ }^{13} \mathrm{C}$ NMR (75.5 MHz, CDCl 3$) 134.4\left(2 \mathrm{sp}^{2}\right.$ tertiary C) 120.8, $40.2\left(\mathrm{CH}_{2}\right), 15.4\left(\mathrm{CH}_{3}\right), 11.0\left(\mathrm{CH}_{3}\right) . \mathrm{IR},(\mathrm{KBr})$ $v / \mathrm{cm}^{-1}: 2980(\mathrm{C}-\mathrm{H}), 1520,1470,1440,1400,1380,1350,1080,1010,760 . \mathrm{m} / \mathrm{z}(\mathrm{EI}) 281\left(\mathrm{M}^{+}, 13\right), 249(2)$, 219 (14), 217 (100), 185 (13), 184 (53), 152 (12). $\mathrm{C}_{8} \mathrm{H}_{11} \mathrm{NS}_{5}$ requires $\left(\mathrm{M}^{+}\right)$280.9495, found $\left(\mathrm{M}^{+}\right) 280.9485$. Consistent with a previous report [61].

7-Methyl-6,8-diphenyl-7H-(1,2,3,4,5)pentathiepino(6,7-c)pyrrole (20) yellow solid (12.1 g, 62\%) m.p. $252-253{ }^{\circ} \mathrm{C}$. Anal. Calcd for $\mathrm{C}_{17} \mathrm{H}_{13} \mathrm{NS}_{5}$ (\%): C, 52.18; H, 3.35; N, 3.58. Found (\%) C, 52.35; H, 3.48; N, 3.22. ${ }^{1} \mathrm{H}$ NMR (300 MHz, $\left.\mathrm{CDCl}_{3}\right)$ 8: $7.46(10 \mathrm{H}, \mathrm{m}, \mathrm{ArH}), 0.34(3 \mathrm{H}, \mathrm{s}, \mathrm{CH} 3),{ }^{13} \mathrm{C}$ NMR $(75.5$ $\left.\mathrm{MHz}, \mathrm{CDCl}_{3}\right) 139.9\left(3 \times \mathrm{sp}^{2}\right.$ tertiary C), 130.3, 131.1, $128.9(3 \times \mathrm{C}-\mathrm{H}), 128.5,125.7,34.8\left(\mathrm{CH}_{3}\right) . \mathrm{IR},(\mathrm{KBr})$ $v / \mathrm{cm}^{-1}:$ 3050, 1470, 1440, 1430, 1380, 1080, 1010, 920, 810, 780, 750, 700. $\mathrm{m} / \mathrm{z}$ (EI) 391 (M $\left.\mathrm{M}^{+}, 20\right), 327$ (100), 295 (52), 280 (15), 118 (53). $\mathrm{C}_{17} \mathrm{H}_{13} \mathrm{NS}_{5}$ requires $\left(\mathrm{M}^{+}\right)$390.9651, found $\left(\mathrm{M}^{+}\right)$390.9669. Consistent with a previous report [61].

6,8-Dichloro-7-methyl-7H-(1,2,3,4,5)pentathiepino(6,7-c)pyrrole (21) yellow solid (5.8 g, 38\%) m.p. 167-170 ${ }^{\circ} \mathrm{C}$. Anal. Calcd for $\mathrm{C}_{5} \mathrm{H}_{3} \mathrm{Cl}_{2} \mathrm{NS}_{5}(\%)$ : $\mathrm{C}, 19.5 ; \mathrm{H}, 1.0 ; \mathrm{N}, 4.5$. Found (\%) $\mathrm{C}, 19.8 ; \mathrm{H}, 1.2 ; \mathrm{N}, 4.2$. ${ }^{1} \mathrm{H}$ NMR $\left(300 \mathrm{MHz}, \mathrm{CDCl}_{3}\right) \delta: 3.60\left(\mathrm{~s}, 3 \mathrm{H}, \mathrm{CH}_{3}\right) ;{ }^{13} \mathrm{C} \mathrm{NMR}\left(75.5 \mathrm{MHz}, \mathrm{CDCl}_{3}\right) 123.8$ and $121.5\left(2 \times \mathrm{sp}^{2}\right.$ tertiary C), $33.2\left(\mathrm{CH}_{3}\right)$. IR, (KBr) v/cm $\mathrm{cm}^{-1}$ : 1460, 1420, 1340, 1100, 780. $\mathrm{m} / \mathrm{z}(\mathrm{EI}) 309\left(\mathrm{M}^{+}+2,6\right), 307\left(\mathrm{M}^{+}\right.$, 9), 245 (39), 243 (48). $\mathrm{C}_{5} \mathrm{H}_{3} \mathrm{Cl}_{2} \mathrm{NS}_{5}$ requires $\left(\mathrm{M}^{+}\right)$306.8246, found $\left(\mathrm{M}^{+}\right)$306.8238. Consistent with a previous report [64]. 
6,8-Dichloro-7-isopropyl-7H-(1,2,3,4,5)pentathiepino(6,7-c)pyrrole (22) yellow solid (6.4 g, 38\%) m.p. $136-139^{\circ} \mathrm{C}$. Anal. Calcd for $\mathrm{C}_{7} \mathrm{H}_{7} \mathrm{Cl}_{2} \mathrm{NS}_{5}(\%)$ : C, 25.0; H, 2.1; N, 4.2. Found (\%) C, 24.7; $\mathrm{H}, 1.9 ; \mathrm{N}$, 4.5. ${ }^{1} \mathrm{H}$ NMR $\left(300 \mathrm{MHz}, \mathrm{CDCl}_{3}\right)$ 8: 5.01 (septet, $\left.1 \mathrm{H}, \mathrm{J}=6.5, \mathrm{CH}\right), 1.61\left(\mathrm{~d}, 6 \mathrm{H}, \mathrm{J}=6.5,2 \mathrm{CH}_{3}\right) ;{ }^{13} \mathrm{C} \mathrm{NMR}$ $\left(75.5 \mathrm{MHz}, \mathrm{CDCl}_{3}\right) 115.3$ and $78.1\left(2 \times \mathrm{sp}^{2}\right.$ tertiary C), $52.1(\mathrm{CH}), 21.7\left(\mathrm{CH}_{3}\right) . \mathrm{IR},(\mathrm{KBr}) \mathrm{v} / \mathrm{cm}^{-1}: 2980$ (CH), 1480, 1400, 1290, 1180, 1140, $795(\mathrm{C}-\mathrm{Cl}) ; \mathrm{m} / z(\mathrm{EI}) 339\left(\mathrm{M}^{+}+4,1 \%\right), 337\left(\mathrm{M}^{+}+2,3 \%\right), 303(15), 301$ (22), 273 (17), 271 (22), 237 (85), 195 (100). $\mathrm{C}_{7} \mathrm{H}_{7} \mathrm{Cl}_{2} \mathrm{NS}_{5}$ requires $\left(\mathrm{M}^{+}\right) 334.8559$, found $\left(\mathrm{M}^{+}\right) 334.8550$. Consistent with a previous report [64].

\subsubsection{7-Chloro-6-methyl-6H-(1,2,3,4,5)pentathiepino(6,7-b)pyrrole (23)}

Sulfur monochloride $(1.6 \mathrm{~mL}, 20 \mathrm{mmol})$ was added dropwise at $-30{ }^{\circ} \mathrm{C}$ to $-35{ }^{\circ} \mathrm{C}$ to a stirred solution of $\mathrm{N}$-methylpyrrole $(0.40 \mathrm{~g}, 5.0 \mathrm{mmol})$ and DABCO $(2.24 \mathrm{~g}, 20 \mathrm{mmol})$ dissolved in chloroform $(50 \mathrm{~mL})$. Then, the mixture was stirred for $15 \mathrm{~min}$ at $-20^{\circ} \mathrm{C}$ and at room temperature for $48 \mathrm{~h}$. The solvent was removed under reduced pressure. The residue was separated by column chromatography (light petroleum, and then light petroleum- $\mathrm{CH}_{2} \mathrm{Cl}_{2}$ mixtures) to produce a yellow solid $(0.518 \mathrm{~g}$, 1.9 mmol, 38\%) m.p. $68-69{ }^{\circ} \mathrm{C}$. Anal. Calcd for $\mathrm{C}_{5} \mathrm{H}_{4} \mathrm{ClNS}_{5}$ (\%): $\mathrm{C}, 21.93 ; \mathrm{H}, 1.47 ; \mathrm{N}, 5.11$. Found (\%) $\mathrm{C}$, 22.08; $\mathrm{H}, 1.56 ; \mathrm{N}, 5.23 .{ }^{1} \mathrm{H}$ NMR $\left(300 \mathrm{MHz}, \mathrm{CDCl}_{3}\right) \delta: 3.71\left(\mathrm{~s}, 3 \mathrm{H}, \mathrm{CH}_{3}\right), 6.43(\mathrm{~s}, 1 \mathrm{H}, \mathrm{pyr}) ;{ }^{13} \mathrm{C} \mathrm{NMR}$ $\left(75.5 \mathrm{MHz}, \mathrm{CDCl}_{3}\right) 33.2\left(\mathrm{CH}_{3}\right), 113.7(\mathrm{CH}), 118.8,128.2$ and $132.1\left(3 \mathrm{sp}^{2}\right.$ tertiary $\left.\mathrm{C}\right) ; \mathrm{m} / \mathrm{z}(\mathrm{EI}) 275\left(\mathrm{M}^{+}\right.$ $+2,13 \%), 273\left(\mathrm{M}^{+}, 24 \%, 209\left(\mathrm{M}-\mathrm{S}_{2}, 100\right) . \mathrm{C}_{5} \mathrm{H}_{4} \mathrm{ClNS}_{5}\right.$ requires $\left(\mathrm{M}^{+}\right) 272.8636$, found $\left(\mathrm{M}^{+}\right) 272.8643$. Consistent with a previous report [32].

\subsubsection{6-Methyl-6H-(1,2,3,4,5)pentathiepino(6,7-b)indole (24)}

Disulfur dichloride $(1.2 \mathrm{~mL}, 20 \mathrm{mmol})$ was added dropwise at -30 to $-35^{\circ} \mathrm{C}$ to a stirred solution of 1-methylindole $(2.55 \mathrm{~g}, 19.5 \mathrm{mmol})$ dissolved in chloroform $(50 \mathrm{~mL})$. Then the mixture was stirred for $15 \mathrm{~min}$ at $-20^{\circ} \mathrm{C}$ and at $0{ }^{\circ} \mathrm{C}$ for $48 \mathrm{~h}$. The mixture was refluxed for $3 \mathrm{~h}$, filtered, and the solvent was removed under reduced pressure. The residue was separated by column chromatography (light petroleum, and then light petroleum- $\mathrm{CH}_{2} \mathrm{Cl}_{2}$ mixtures) to produce a yellow solid $(0.520 \mathrm{~g}, 70 \%) \mathrm{m} . \mathrm{p}$. 123-125 ${ }^{\circ} \mathrm{C}$. Anal. Calcd for $\mathrm{C}_{9} \mathrm{H}_{7} \mathrm{NS}_{5}(\%): 37.3 ; \mathrm{H}, 2.4 ; \mathrm{N}, 4.8$. Found $(\%) \mathrm{C}, 37.5 ; \mathrm{H}, 2.3 ; \mathrm{N}, 4.9 .{ }^{1} \mathrm{H}$ NMR $\left(300 \mathrm{MHz}, \mathrm{CDCl}_{3}\right)$ 8: $7.70(\mathrm{~m}, 1 \mathrm{H}, \mathrm{PhH}), 7.30(\mathrm{~m}, 1 \mathrm{H}, \mathrm{PhH}), 3.91\left(\mathrm{~s}, 3 \mathrm{H}, \mathrm{CH}_{3}\right) .{ }^{13} \mathrm{C} \mathrm{NMR}(75.5$ $\left.\mathrm{MHz}, \mathrm{CDCl}_{3}\right) 141.8,137.1,129.5$ and $126.0\left(4 \times \mathrm{sp}^{2}\right.$ tertiary $\left.\mathrm{C}\right), 125.2,122.7,119.6$ and $111.1(4 \times \mathrm{CH})$, $32.1\left(\mathrm{CH}_{3}\right)$. IR, (KBr) v/cm ${ }^{-1}: 2980(\mathrm{CH}), 1440,1330,1240,1160,820,780,740 \mathrm{~m} / \mathrm{z}(\mathrm{EI}) 289\left(\mathrm{M}^{+}, 22 \%\right)$, 225 (100), 192 (42). Consistent with a previous report [64].

Author Contributions: Conceptualization, C.R.M.A. and L.H.M.d.M.; methodology, C.R.M.A., A.C.S.M., L.H.M.d.M., L.S.K., R.A.-P., O.A.R. and S.A.P.; formal analysis, C.R.M.A., A.C.S.M., L.H.M.d.M. and L.S.K.; investigation, C.R.M.A., A.C.S.M., L.H.M.d.M. and L.S.K.; resources, C.R.M.A., R.A., O.A.R. and S.A.P.; data curation, C.R.M.A., A.C.S.M., L.H.M.d.M., L.S.K.; writing-original draft preparation, C.R.M.A.; writing-review and editing, C.R.M.A., A.C.S.M., L.H.M.d.M., L.S.K., R.A., O.A.R. and S.A.P.

Funding: The authors are grateful to Fundação Carlos Chagas Filho de Amparo à Pesquisa do Estado do Rio de Janeiro (FAPERJ/JCNE, grant no. E-26/202.737/2019), Conselho Nacional de Pesquisa e Desenvolvimento Tecnológico (CNPq) (Grant no. 305487/2015-9) and Russian Science Foundation (grant no. 15-13-10022) for financial support towards the goals of our work. S.A.P. is the recipient of a productivity fellowship from Conselho Nacional de Pesquisa e Desenvolvimento Tecnológico (CNPq).

Acknowledgments: The authors thank Anthony J. Hickey and Tammy M. Havener (both UNC Catalyst for Rare Diseases, University of North Carolina at Chapel Hill) for proof reading the manuscript and useful discussions.

Conflicts of Interest: The authors declare no conflict of interest.

\section{References}

1. Dias, N.M.; Oliveira, M.M.; Santos, C.; Zancope-Oliveira, R.M.; Lima, N. Sporotrichosis caused by Sporothrix Mexicana, Portugal. Emerg. Infect. Dis. 2011, 17, 1975-1976. [CrossRef] [PubMed]

2. Schenck, B.R. On refractory subcutaneous abscesses casued by a fungus possibly related to the sporotricha. Bull. Johns Hopkins Hosp. 1898, 93, 286. 
3. Gremião, I.D.; Miranda, L.H.; Reis, E.G.; Rodrigues, A.M.; Pereira, S.A. Zoonotic epidemic of sporotrichosis: Cat to human transmission. PLoS Pathog. 2017, 13, e1006077. [CrossRef] [PubMed]

4. Barros, M.B.; Schubach Ade, O.; do Valle, A.C.; Gutierrez Galhardo, M.C.; Conceição-Silva, F.; Schubach, T.M.; Reis, R.S.; Wanke, B.; Marzochi, K.B.; Conceição, M. Cat-transmitted sporotrichosis epidemic in Rio de Janeiro, Brazil: Description of a series of cases. J. Clin. Infect. Dis. 2004, 38, 529-535. [CrossRef]

5. Almeida-Paes, R.; de Oliveira, M.M.; Freitas, D.F.; do Valle, A.C.; Zancopé-Oliveira, R.M.; Gutierrez-Galhardo, M.C. Sporotrichosis in Rio de Janeiro, Brazil: Sporothrix brasiliensis is associated with atypical clinical presentations. PLoS Negl. Trop. Dis. 2014, 8, e3094. [CrossRef]

6. Barros, M.B.; de Almeida Paes, R.; Schubach, A.O. Sporothrix schenckii and Sporotrichosis. Clin. Microbiol. Rev. 2011, 24, 633-654. [CrossRef]

7. van Burik, J.A.; Magee, P.T. Aspects of fungal pathogenesis in humans. Annu. Rev. Microbiol. 2001, 55, 743-772. [CrossRef]

8. Chakrabarti, A.; Bonifaz, A.; Gutierrez-Galhardo, M.C.; Mochizuki, T.; Li, S. Global epidemiology of sporotrichosis. Med. Mycol. 2015, 53, 3-14. [CrossRef]

9. Rodrigues, A.M.; de Hoog, G.S.; de Camargo, Z.P. Sporothrix species causing outbreaks in animals and humans driven by animal-animal transmission. PLoS Pathog. 2016, 12, e1005638. [CrossRef]

10. Gremião, I.D.F.; Menezes, R.C.; Schubach, T.M.P.; Figueiredo, A.B.F.; Cavalcanti, M.C.; Pereira, S.A. Feline sporotrichosis: Epidemiological and clinical aspects. Med. Mycol. 2015, 53, 15-21. [CrossRef]

11. Falcão, E.M.M.; Pires, M.C.S.; Andrade, H.B.; Gonçalves, M.L.C.; Almeida-Paes, R.; do Valle, A.C.F.; Bastos, F.I.; Gutierrez-Galhardo, M.C.; Freitas, D.F.S. Zoonotic sporotrichosis with greater severity in Rio de Janeiro, Brazil: 118 hospitalizations and 11 deaths in the last 2 decades in a reference institution. Med. Mycol. 2019, myz024. [CrossRef] [PubMed]

12. Miranda, L.H.M.; Silva, J.N.; Gremião, I.D.F.; Menezes, R.C.; Almeida-Paes, R.; Dos Reis, É.G.; de Oliveira, R.V.C.; de Araujo, D.S.D.A.; Ferreiro, L.; Pereira, S.A. Monitoring fungal burden and viability of Sporothrix spp. in skin lesions of cats for predicting antifungal treatment response. J. Fungi 2018, 4, 92. [CrossRef] [PubMed]

13. Mazum, T.K.; Bricker, B.A.; Flores-Rozas, H.; Ablordeppey, S.Y. The mechanistic targets of antifungal agents: An overview. Mini Rev. Med. Chem. 2016, 16, 555-578. [CrossRef] [PubMed]

14. López, S.N.; Castelli, M.V.; Zacchino, S.A.; Domínguez, J.N.; Lobo, G.; Charris-Charris, J.; Cortés, J.C.; Ribas, J.C.; Devia, C.; Rodríguez, A.M.; et al. In vitro antifungal evaluation and structure-activity relationships of a new series of chalcone derivatives and synthetic analogues, with inhibitory properties against polymers of the fungal cell wall. Bioorg. Med. Chem. 2001, 9, 1999-2013. [CrossRef]

15. Urbina, J.M.; Cortés, J.C.; Palma, A.; López, S.N.; Zacchino, S.A.; Enriz, R.D.; Ribas, J.C.; Kouznetzov, V.V. Inhibitors of the fungal cell wall. Synthesis of 4-aryl-4-N-arylamine-1-butenes and related compounds with inhibitory activities on beta(1-3) glucan and chitin synthases. Bioorg. Med. Chem. 2000, 8, 691-698. [CrossRef]

16. Vargas, M.L.Y.; Castelli, M.V.; Kouznetsov, V.V.; Urbina, G.J.M.; López, S.N.; Sortino, M.; Enriz, R.D.; Ribas, J.C.; Zacchino, S. In vitro antifungal activity of new series of homoallylamines and related compounds with inhibitory properties of the synthesis of fungal cell wall polymers. Bioorg. Med. Chem. 2003, 11, 1531-1550. [CrossRef]

17. Giannini, F.A.; Aimar, M.L.; Sortino, M.; Gomez, R.; Sturniollo, A.; Juarez, A.; Zacchino, S.; de Rossi, R.H.; Enriz, R.D. In vitro-in vivo antifungal evaluation and structure-activity relationships of 3H-1,2-dithiole-3-thione derivatives. Farmaco 2004, 59, 245-254. [CrossRef]

18. Vladuchick, S.A. Fungicidal Isothiazoles. U.S. Patent 4094985 A, 13 June 1977.

19. Moberg, K. Fungicidal Compounds. U.S. Patent 4275073 A, 23 June 1981.

20. Chenard, B.L. Substituted Benzopentathiepins. U.S. Patent 4571404 A, 18 February 1984.

21. Chenard, B.L. 8-Substituted Pyrazolopentathiepins and Related Compounds. U.S. Patent EP 0138622 A3, 24 April 1985.

22. Vladuchick, S.A.; Fukunaga, T.; Simmons, H.E.; Webster, O.W. Thiacyanocarbons. 6. 1, 4-Dithiino [2, 3-c; 6, 5-c'] diisothiazole-3, 7-dicarbonitrile, isothiazole [3, 4-f][1, 2, 3, 4, 5] pentathiepine-8-carbonitrille, and disodium 5-cyanoisothiazoledithiolate. J. Org. Chem. 1980, 45, 5122-5130. [CrossRef]

23. Chenard, B.L.; Miller, T.J. Benzopentathiepins: Synthesis via thermolysis of benzothiadiazoles with sulfur. J. Org. Chem. 1984, 49, 1221-1224. [CrossRef] 
24. Van Cutsem, J.; Van Gerven, F.; Fransen, J.; Schrooten, P.; Janssen, P.A. The in vitro antifungal activity of ketoconazole, zinc pyrithione, and selenium sulfide against Pityrosporum and their efficacy as a shampoo in the treatment of experimental pityrosporosis in guinea pigs. J. Am. Acad. Dermatol. 1990, 22, 993-998. [CrossRef]

25. Cohen, P.R.; Anderson, C.A. Topical selenium sulfide for the treatment of Hyperkeratosis. Dermatol. Ther. 2018, 8, 639-646. [CrossRef] [PubMed]

26. Pekonen, P.; Hiltunen, Y.; Laitinen, R.S.; Pakkanen, T.A. Selenium-77 NMR spectroscopic and X-ray crystallographic characterization of bis(cyclopentadienyl)titanium selenide sulfide mixtures [Ti(C5H5)2SexS5x]. Inorg. Chem. 1991, 30, 3679. [CrossRef]

27. Chandler, C.J.; Segel, I.H. Mechanism of the antimicrobial action of pyrithione: Effects on membrane transport, ATP levels, and protein synthesis. Antimicrob. Agents Chemother. 1978, 14, 60-68. [CrossRef] [PubMed]

28. Feng, M.; Tang, B.; Liang, S.H.; Jiang, X. Sulfur containing scaffolds in drugs: Synthesis and application in medicinal chemistry. Curr. Top. Med. Chem. 2016, 16, 1200-1216. [CrossRef] [PubMed]

29. Asquith, C.R.M.; Meli, M.L.; Konstantinova, L.S.; Laitinen, T.; Peräkylä, M.; Poso, A.; Rakitin, O.A.; Allenspach, K.; Hofmann-Lehmann, R.; Hilton, S.T. Evaluation of the antiviral efficacy of bis[1,2]dithiolo[1,4]thiazines and bis[1,2]dithiolopyrrole derivatives against the nucelocapsid protein of the Feline Immunodeficiency Virus (FIV) as a model for HIV infection. Bioorg. Med. Chem. Lett. 2014, 24, 2640-2644. [CrossRef] [PubMed]

30. Asquith, C.R.M.; Meli, M.L.; Konstantinova, L.S.; Laitinen, T.; Poso, A.; Rakitin, O.A.; Hofmann-Lehmann, R.; Allenspach, K.; Hilton, S.T. Novel fused tetrathiocines as antivirals that target the nucleocapsid zinc finger containing protein of the feline immunodeficiency virus (FIV) as a model of HIV infection. Bioorg. Med. Chem. Lett. 2015, 25, 1352-1355. [CrossRef]

31. Asquith, C.R.M.; Konstantinova, L.S.; Meli, M.L.; Laitinen, T.; Poso, A.; Rakitin, O.A.; Hofmann-Lehmann, R.; Hilton, S.T. Evaluation of substituted 1,2,3-Dithiazoles as inhibitors of the feline immunodeficiency virus (FIV) nucleocapsid protein via a proposed Zinc ejection mechanism. ChemMedChem 2016, 11, 2119-2126. [CrossRef]

32. Asquith, C.R.M.; Laitinen, T.; Konstantinova, L.S.; Poso, A.; Rakitin, O.A.; Hofmann-Lehmann, R.; Hilton, S.T. Investigation of the pentathiepin functionality as an inhibitor of feline immunodeficiency virus (FIV) via a potential zinc ejection mechanism, as a model for HIV infection. ChemMedChem 2019, 14, 454-461. [CrossRef]

33. Asquith, C.R.M.; Meili, T.; Laitinen, T.; Baranovsky, I.V.; Konstantinova, L.S.; Poso, A.; Rakitin, O.A.; Hofmann-Lehmann, R. Synthesis and comparison of substituted 1,2,3-dithiazole and 1,2,3-thiaselenazole as inhibitors of the feline immunodeficiency virus (FIV) nucleocapsid protein as a model for HIV infection. Bioorg. Med. Chem. Lett. 2019, 29, 1765-1768. [CrossRef]

34. Loo, J.A.; Holler, T.P.; Sanchez, J.; Gogliotti, R.; Maloney, L.; Reily, M.D. Biophysical characterization of zinc ejection from HIV nucleocapsid protein by anti-HIV 2,2'-dithiobis[benzamides] and benzisothiazolones. $J$. Med. Chem. 1996, 39, 4313-4320. [CrossRef]

35. Chen, S.C.; Jeng, K.S.; Lai, M.M.C. Zinc finger-containing cellular transcription corepressor ZBTB25 promotes influenza virus RNA transcription and is a target for zinc ejector drugs. J. Virol. 2017, 91, e00842-17. [CrossRef] [PubMed]

36. Sekirnik, R.; Rose, N.R.; Thalhammer, A.; Seden, P.T.; Mecinović, J.; Schofield, C.J. Inhibition of the histone lysine demethylase JMJD2A by ejection of structural Zn(II). Chem. Commun. 2009, 42, 6376-6378. [CrossRef] [PubMed]

37. Cook, K.M.; Hilton, S.T.; Mecinović, J.; Motherwell, W.B.; Figg, W.D.; Schofield, C.J. Epidithiodiketopiperazines block the interaction between hypoxia-inducible factor-1alpha (HIF-1alpha) and p300 by a zinc ejection mechanism. J. Biol. Chem. 2009, 284, 26831-26838. [CrossRef] [PubMed]

38. Subramanian Vignesh, K.; Deepe, G.S., Jr. Metallothioneins: Emerging modulators in immunity and infection. Int. J. Mol. Sci. 2017, 18, 2197. [CrossRef]

39. Davidson, B.S.; Molinski, T.F.; Barrows, L.R.; Ireland, C.M. Varacin: A novel benzopentathiepin from Lissoclinum vareau that is cytotoxic toward a human colon tumor. J. Am. Chem. Soc. 1991, 113, 4709-4710. [CrossRef]

40. Litaudon, M.; Trigalo, F.; Martin, M.; Frappier, F.; Guyot, M. Lissoclinotoxins: Antibiotic polysulfur derivatives from the tunicate Lissoclinum perforatum. Revised structure of lissoclinotoxin A. Tetrahedron 1994, 50, 5323. [CrossRef] 
41. Lee, A.H.; Chan, A.S.; Li, T. Acid-accelerated DNA-cleaving activities of antitumor antibiotic varacin. Chem. Commun. 2002, 18, 2112. [CrossRef]

42. Compagnone, R.S.; Faulkner, D.J.; Carté, B.K.; Chan, G.; Freyer, A.; Hemling, M.E.; Hofmann, G.A.; Mattern, M.R. Pentathiepins and trithianes from two Lissoclinum species and a Eudistoma sp.: Inhibitors of protein kinase C. Tetrahedron 1994, 50, 12785. [CrossRef]

43. Sato, R.; Ohyama, T.; Ogawa, S. Efficient synthesis and biological properties of new benzopentathiepins. Heterocycles 1995, 41, 893-896. [CrossRef]

44. Chatterji, T.; Gates, K.S. DNA cleavage by 7-methylbenzopentathiepin: A simple analog of the antitumor antibiotic varacin. Bioorg. Med. Chem. Lett. 1998, 8, 535-538. [CrossRef]

45. Chatterji, T.; Gates, K.S. Reaction of thiols with 7-methylbenzopentathiepin. Bioorg. Med. Chem. Lett. 2003, 13, 1349-1352. [CrossRef]

46. Liu, H.; Fujiwara, T.; Nishikawa, T.; Mishima, Y.; Nagai, H.; Shida, T.; Tachibana, K.; Kobayashi, H.; Mangindaan, R.E.P.; Namikoshi, M. Lissoclibadins 1-3, three new polysulfur alkaloids, from the ascidian Lissoclinum cf. badium. Tetrahedron 2005, 61, 8611-8615. [CrossRef]

47. Baguley, T.D.; Nairn, A.C.; Lombroso, P.J.; Ellman, J.A. Synthesis of benzopentathiepin analogs and their evaluation as inhibitors of the phosphatase STEP. Bioorg. Med. Chem. Lett. 2015, 25, 1044-1046. [CrossRef] [PubMed]

48. Xu, J.; Chatterjee, M.; Baguley, T.D.; Brouillette, J.; Kurup, P.; Ghosh, D.; Kanyo, J.; Zhang, Y.; Seyb, K.; Ononenyi, C.; et al. Inhibitor of the tyrosine phosphatase STEP reverses cognitive deficits in a mouse model of Alzheimer's disease. PLoS Biol. 2014, 12, e1001923. [CrossRef] [PubMed]

49. Zakharenko, A.; Khomenko, T.; Zhukova, S.; Koval, O.; Zakharova, O.; Anarbaev, R.; Lebedeva, N.; Korchagina, D.; Komarova, N.; Vasiliev, V.; et al. Synthesis and biological evaluation of novel tyrosyl-DNA phosphodiesterase 1 inhibitors with a benzopentathiepine moiety. Bioorg. Med. Chem. 2015, 23, $2044-2052$. [CrossRef] [PubMed]

50. Khomenko, T.M.; Korchagina, D.V.; Baev, D.S.; Vassiliev, P.M.; Volcho, K.P.; Salakhutdinov, N.F. Antimicrobial activity of substituted benzopentathiepin-6-amines. J. Antibiot. 2019, 72, 590-599. [CrossRef]

51. Clinical and Laboratory Standards Institute (CLSI). Reference Method for Broth Dilution Antifungal Susceptibility Testing of Filamentous Fungi, 2nd ed.; Clinical and Laboratory Standards Institute: Wayne, MI, USA, 2008; p. 52.

52. Almeida-Paes, R.; Brito-Santos, F.; Figueiredo-Carvalho, M.H.G.; Machado, A.C.S.; Oliveira, M.M.E.; Pereira, S.A.; Gutierrez-Galhardo, M.C.; Zancopé-Oliveira, R.M. Minimal inhibitory concentration distributions and epidemiological cutoff values of five antifungal agents against Sporothrix brasiliensis. Memórias do Instituto Oswaldo Cruz 2017, 112, 376-381. [CrossRef]

53. Fehér, F.; Degen, B. New Sulfur-containing Cyclic Compounds. Angew. Chem. Int. Ed. 1967, 6, 703-704. [CrossRef]

54. Fehér, F.; Langer, M. Contribution to the chemistry of sulfur, no. 104 Synthesis of pentathiepin and benzopentathiepin. Tetrahedron Lett. 1971, 12, 2125-2126. [CrossRef]

55. Fehér, F.; Langer, M.; Volkert, R.Z. Contributions to the chemistry of sulfur, 112. Naturforsch. B 1972, $27,1006$. [CrossRef]

56. Konstantinova, L.S.; Rakitin, O.A.; Rees, C.W. Pentathiepins. Chem. Rev. 2004, 104, 2617-2630. [CrossRef] [PubMed]

57. Konstantinova, L.S.; Amelichev, S.A.; Rakitin, O.A. 1,2,3,4,5-Pentathiepines and 1,2,3,4,5-pentathiepanes. Russ. Chem. Rev. 2007, 76, 195-211. [CrossRef]

58. Konstantinova, L.S.; Rakitin, O.A. Design of sulfur heterocycles with sulfur monochloride: Synthetic possibilities and prospects. Mendeleev Commun. 2009, 19, 55-61. [CrossRef]

59. Rakitin, O.A.; Konstantinova, L.S. Chapter 4 Sulfur Monochloride in the Synthesis of Heterocyclic Compounds. Adv. Heterocycl. Chem. 2008, 96, 175-229.

60. Konstantinova, L.S.; Rakitin, O.A.; Rees, C.W.; Souvorova, L.I.; Golovanov, D.G.; Lyssenko, K.A. Unprecedented conversion of triethylamine and disulfur dichloride into a thienopentathiepin and a heptathiocane. Org. Lett. 2003, 5, 1939-1942. [CrossRef]

61. Amelichev, S.A.; Aysin, R.R.; Konstantinova, L.S.; Obruchnikova, N.V.; Rakitin, O.A.; Rees, C.W. Abnormally mild synthesis of bis(dithiolo)pyrroles from 2,5-dimethylpyrroles. Org. Lett. 2005, 7, 5725-5727. [CrossRef] 
62. Konstantinova, L.S.; Amelichev, S.A.; Rakitin, O.A. Regioselective synthesis of pentathiepines fused with pyrrole, thiophene, or indole rings. Russ. Chem. Bull. 2006, 55, 2081-2084. [CrossRef]

63. Konstantinova, L.S.; Rakitin, O.A.; Rees, C.W.; Amelichev, S.A. Regioselective synthesis of pentathiepino-fused pyrroles and indoles. Mendeleev Commun. 2004, 14, 91-92. [CrossRef]

64. Amelichev, S.A.; Konstantinova, L.S.; Lyssenko, K.A.; Rakitin, O.A.; Rees, C.W. Direct synthesis of fused 1,2,3,4,5-pentathiepins. Org. Biomol. Chem. 2005, 3, 3496-3501. [CrossRef]

65. Konstantinova, L.S.; Rakitin, O.A.; Rees, C.W. A one-step synthesis of fused pentathiepins. Chem. Comm. 2002, 11, 1204-1205. [CrossRef]

66. Boechat, J.S.; Oliveira, M.M.E.; Almeida-Paes, R.; Gremião, I.D.F.; Machado, A.C.S.; Oliveira, R.V.C.; Figueiredo, A.B.F.; Rabello, V.B.S.; Silva, K.B.L.; Zancopé-Oliveira, R.M.; et al. Feline sporotrichosis: Associations between clinical-epidemiological profiles and phenotypic-genotypic characteristics of the etiological agents in the Rio de Janeiro epizootic area. Memórias do Instituto Oswaldo Cruz 2018, 113, 185-196. [CrossRef] [PubMed]

(C) 2019 by the authors. Licensee MDPI, Basel, Switzerland. This article is an open access article distributed under the terms and conditions of the Creative Commons Attribution (CC BY) license (http://creativecommons.org/licenses/by/4.0/). 